\title{
Deviations from early-time quasilinear behaviour for the quantum kicked rotor near the classical limit
}

\author{
Mark Sadgrove, Terry Mullins, Scott Parkins, and Rainer Leonhardt \\ Department of Physics, The University of Auckland, Private Bag 92019, Auckland, New Zealand
}

\begin{abstract}
We present experimental measurements of the mean energy for the atom optics kicked rotor after just two kicks. The energy is found to deviate from the quasi-linear value for small kicking periods. The observed deviation is explained by recent theoretical results which include the effect of a nonuniform initial momentum distribution, previously applied only to systems using much colder atoms than ours.
\end{abstract}

PACS numbers: 5.45.-a, 42.50.Vk

For some time now, studies of cold atoms subject to a periodically pulsed 1D optical lattice (a system referred to as the atom optics kicked rotor (AOKR)) have provided a great deal of insight into quantum systems with chaotic classical analogues 1, 2, 3, 4]. Typically, such experiments have focussed on the "late time" behaviour of the quantum kicked rotor. This is partially because the hallmark of quantum interference effects in the AOKR dynamical localisation - is only observed after at least five or ten kicks for typical experimental parameters (see for example [5]). Observations of quantum resonances in the mean energy have also generally been made in the late time regime [4, 6]. Somewhat less attention hasbeen given, however, to the "early time" regime, where theoretical predictions have suggested that only quasilinear energy growth takes place, in particular, during the first two kicks. That is, no classical or quantum correlations are expected to be important during this time, given broad initial distributions for position and momentum.

However, recent theoretical and experimental work demonstrates that non-trivial behaviour may be observed in the mean energy of the AOKR as a function of kicking period after as few as two kicks if the atomic sample initially has a narrow momentum distribution [7]. Experimental work has focussed on the temporal half Talbot effect which leads to recurrence of the initial momentum distribution after two kicks [ 8, [9] and more detailed studies of the energy dependence as the kicking period is varied have also been performed [9]. To observe the effects of interest, both of these previous experimental studies made use of Bose-Einstein condensates which provide atomic samples with much narrower initial momentum distributions than those used in typical AOKR experiments. For atomic ensembles with larger thermal energies, the classical theory of Rechester and White 10] and the quantum theory of Shepelyansky [11] both predict the same constant quasi-linear energy growth rate for the first two kicks for any kicking period. In this report we demonstrate that deviations from quasi-linear behaviour can occur in the second kick even for relatively broad initial momentum distributions. The anomalous energy growth rates are found only at very small values of the kicking period which have not previously been probed experimentally.

For large detunings between the kicking laser and the atomic transition, the scaled Hamiltonian for an atom which experiences ideal $\delta$ kicks with period $T$ is

$$
\hat{H}=\frac{\hat{\rho}^{2}}{2}-\kappa \cos (\hat{\phi}) \sum_{n=0}^{N} \delta(\tau-n),
$$

where $\hat{\rho}$ is the scaled atomic momentum operator, $\hat{\phi}$ is the scaled position operator for an atom, $\tau=t / T$ is the scaled time and $\kappa$ is the kicking strength. We note the commutator relation $[\hat{\phi}, \hat{\rho}]=i \hbar$ where $\hbar=8 \omega_{r} T$, and $\hbar \omega_{r}$ is the energy change of a Caesium atom after the scattering of a single photon of wavelength $2 \pi / k_{l}=$ $852 \mathrm{~nm}$ has occurred. The atomic momentum $p$ is related to the scaled momentum $\rho$ by $p / 2 \hbar k_{l}=\rho / \hbar$, and we refer to the quantity $p / 2 \hbar k_{l}$ as the momentum in 2 -photon recoils, or the momentum in experimental units. The atomic position operator $\hat{x}$ is given by $\hat{x}=\hat{\phi} / 2 k_{l}$.

It is useful to consider the standard map for the $\delta$ kicked rotor (DKR). If we label the atomic position and momentum just before the $n$th kick as $\phi_{n-1}$ and $\rho_{n-1}$ respectively, integrating Hamilton's equations over one kick gives the recursive relation:

$$
\begin{aligned}
& \phi_{n}=\phi_{n-1}+\rho_{n} \\
& \rho_{n}=\rho_{n-1}-\kappa \sin \left(\phi_{n-1}\right) .
\end{aligned}
$$

This map holds for the classical position and momentum for the DKR and also for the associated position and momentum operators for the quantum DKR. In this report, we are interested in the mean energy of the atoms after they have experienced two kicks. From Eq. 2 the atomic momentum after two kicks is

$$
\rho_{2}=\rho_{0}-\kappa \sin \left(\phi_{0}\right)-\kappa \sin \left(\phi_{1}\right) .
$$

Experimentally, we measure the quantity $E_{2}=\left\langle\rho_{2}^{2} / 2 k^{2}\right\rangle$, that is, the mean kinetic energy of the atomic ensemble (in experimental units). Theoretically, this requires the determination of correlation functions of the form $\left\langle\sin \left(\phi_{0}\right) \sin \left(\phi_{i}\right)\right\rangle$. These correlations were first calculated for the classical DKR by Rechester and White 10] and later for the quantum DKR by Shepelyansky [11] under 
the assumption that the atomic position and momentum were initially uniformly distributed. In this case, the cross terms in the expression for $\left\langle\rho_{2}^{2}\right\rangle$ are found to vanish

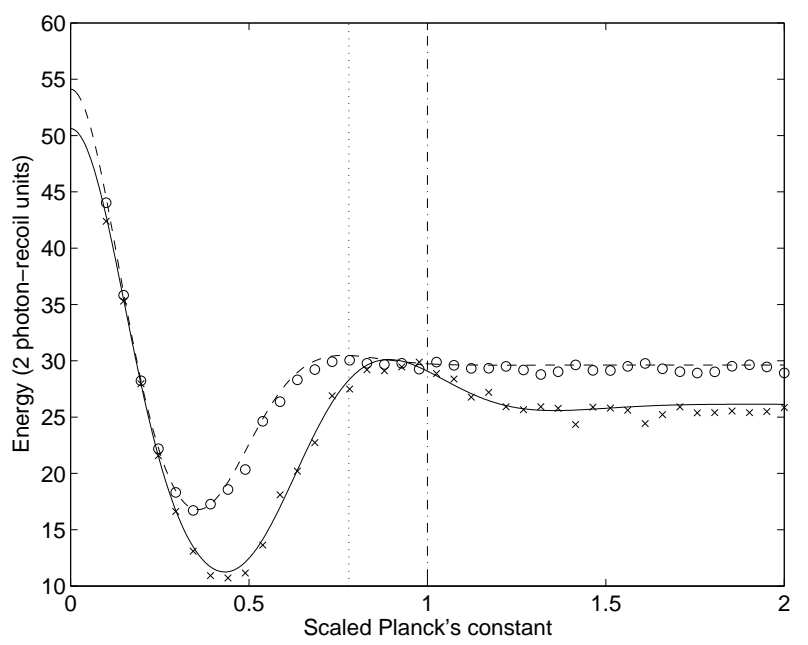

FIG. 1: Energy after 2 kicks as a function of $\hbar$ as given by Eq. 5] The solid line is for $\kappa / \hbar=7$ and $\sigma_{p}=1.82$-photon recoils. The dashed line is for $\kappa / \hbar=7$ and $\sigma_{p}=3.22$-photon recoils. Simulation results for the same parameters (crosses and circles respectively), but for kicking with rectangular pulses of constant width $\tau_{p}$, are overlayed. The ratio $\tau_{p} / T$ varied from about 0.015 for $k \approx 3$ to 0.5 for $k \approx 0.1$. Vertical lines show the value of $k_{\text {crit }}$ as determined from Eq. 6] The dot-dashed line is for $\sigma_{p}=1.8$, and the dotted line is for $\sigma_{p}=3.2$.

when averaged over the ensemble and we are left only with contributions from the squares of the three terms in Eq. 3. The last two terms give equal contributions of $\kappa^{2} / 4$ each, so the energy after two kicks is

$$
E_{2, \text { broad }}=\frac{1}{2 k^{2}}\left(\sigma_{\rho}^{2}+\kappa^{2}\right)
$$

where $\sigma_{\rho}$ is the scaled standard deviation of the initial momentum distribution. Although this expression is explicitly dependent on $k$, in a given experimental run, the quantities $\sigma_{\rho} / \hbar$ and $\kappa / \hbar$ are held constant (corresponding to a constant MOT temperature and constant laser power respectively). In this case, we see that the energy after two kicks remains constant as $k$ is varied and that, ignoring the thermal energy $\sigma_{\rho}^{2} / 2 k^{2}$, it is simply given by twice the quasi-linear growth rate $\kappa^{2} / 4 \hbar^{2}$.

The assumption of a broad initial momentum distribution is not justified if the initial momentum distribution is comparable in width to $2 \hbar k_{l}$ (as is the case for a Bose-Einstein condensate), or if $\hbar$ is close to zero, since $\sigma_{\rho}=\hbar \sigma_{p}$ (where $\sigma_{p}$ is the standard deviation in experimental units). In this report we consider the latter case and the effect it has on the energy after two kicks. In Ref. 7] an expression was derived for the energy after the second kick for atoms with a Gaussian initial momentum distribution of arbitrary width $\sigma_{\rho}$. In this case the cross terms in the expression for $\left\langle\rho_{2}^{2}\right\rangle$ do not vanish and

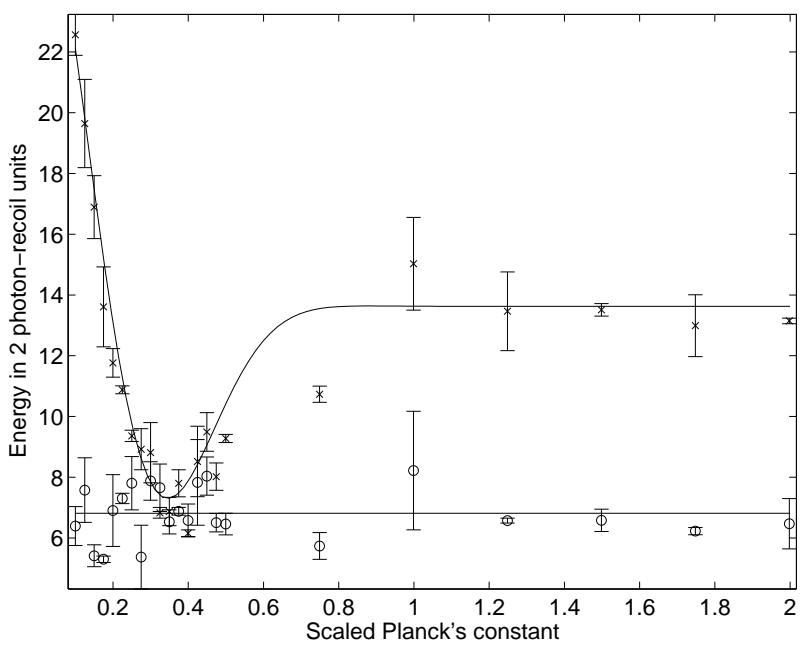

FIG. 2: Experimentally measured energies after one and two kicks (circles and crosses respectively). The value of $\kappa / \hbar$ was found to be $5.2 \pm 0.4$ and $\sigma_{p}$ was measured to be 4.22 -photon recoils. Solid lines give the theoretical values for the energy after one and two kicks respectively. The initial thermal energy has been subtracted to allow a more instructive comparison between the energies.

the energy after two kicks for the quantum DKR is found to be

$$
\begin{aligned}
E_{2}= & \frac{1}{2 k^{2}}\left(\sigma_{\rho}^{2}+\frac{\kappa^{2}}{2}+\frac{\kappa^{2}}{2}\left(1-J_{2}\left(\kappa_{2 q}\right) e^{-2 \sigma_{\rho}^{2}}\right)\right. \\
& -\kappa J_{1}\left(\kappa_{q}\right) \sigma_{\rho}^{2} e^{-\sigma_{\rho}^{2} / 2}+\kappa^{2}\left(J_{0}\left(\kappa_{q}\right)\right. \\
& \left.\left.\left.-J_{2}\left(\kappa_{q}\right)\right) \cos (\hbar / 2) e^{-\sigma_{\rho}^{2} / 2}\right)\right)
\end{aligned}
$$

where $\kappa_{q}=2 \kappa \sin (\hbar / 2) / \hbar$ and $\kappa_{2 q}=2 \kappa \sin (\hbar) / \hbar$. The width of the initial momentum distribution becomes narrower in scaled units as $k$ is decreased, leading to deviations from the quasilinear result for $k \lesssim 1$. This effect can be seen in Fig. 1 for two different values of $\sigma_{p}$. The energy now varies as a function of $k$ and exhibits a pronounced minimum, the position of which depends on the exact value of $\kappa / \hbar$. From this minimum, the energy then increases monotonically as $k \rightarrow 0$ to the value $(\kappa / \hbar)^{2}+\sigma_{p}^{2} / 2$ in $2-$ photon recoil energies.

Physically, we can ascribe the importance of the exact momentum variance of the atoms for low $k$ to the short time between pulses in this regime. Essentially, the spread in atomic momentum is not resolved if the time between the first and second kicks is small. For very small $k$, the system's behaviour is similar to the case where the atomic sample starts in an initial momentum eigenstate. We may estimate the value of $\hbar$ below which we expect deviations from quasi-linear behaviour to occur as follows: After one kick, the atoms have a momentum variance $\sigma_{\text {tot }}^{2}=\sigma_{p}^{2}+\sigma_{1}^{2}$, where $\sigma_{1}$, the momentum variance due to the first kick, is $\sigma_{1}^{2}=2(\kappa / k)^{2}\left(\hbar k_{l}\right)^{2}$ (assuming a broad initial position distribution). The shortest time $T_{\text {crit }}$ between pulses for which atoms with momentum 
$|p|=\sigma_{\text {tot }}$ still traverse a full cycle of the standing wave between kicks is given by

$$
T_{\text {crit }}=\frac{\lambda M_{\mathrm{Cs}}}{2 \sigma_{\mathrm{tot}}} .
$$

For pulsing periods $T<T_{\text {crit }}$ (and, thus, $\hbar<8 \omega_{r} T_{\text {crit }}=$ $\left.k_{\text {crit }}\right)$, the majority of the atoms traverse a distance less than $\lambda / 2$ and deviations from the quasilinear result should be expected. For the two sets of parameters considered in Fig. 11 $k_{\text {crit }}=1.0$ and 0.78 , in good agreement with the behaviour exhibited by numerical and analytical results.

The crosses and circles in Fig. 1 show simulation results for a rectangular-pulse-kicked rotor, in which the pulse width $\tau_{p}$ was kept constant as the kicking period was decreased, as in experiments. We see that even though the $\delta$-kicked approximation is flagrantly violated for low $\hbar$ (where the pulse may be on for up to half the kicking period), the simulation results show excellent agreement with the $\delta$-kicked theory. This agreement may be attributed to the fact that the atomic momenta are still sufficiently small after two kicks, that atoms will not tend to traverse a significant distance along the optical standing wave during the time the pulse is on. Specifically, for the highest energies measured in this work, atoms typically travel a distance of only $8 \%$ of the standing wave's period. This means that averaging of the kicking strength over the pulse duration, which can restrict energy growth after many pulses, may be neglected in this work. Hence, we compare our experimental results with Eq. [5 in the remainder of this report.

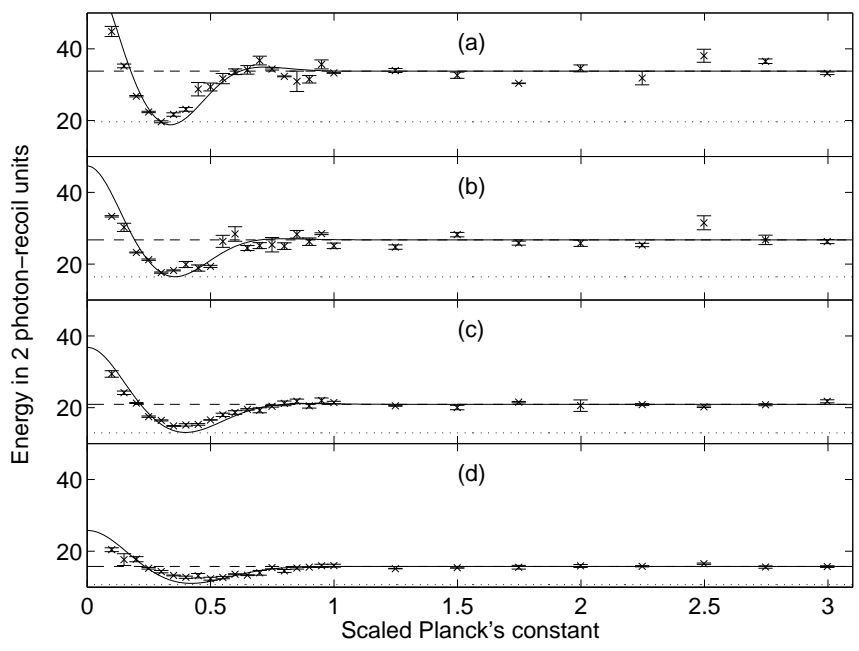

FIG. 3: Experimentally measured energies after two kicks for various values of $\kappa / \hbar$ and $\sigma_{p}=3.35 \pm 0.06$. The measured values of $\kappa / \hbar$ were (a) 7.5 , (b) 6.4 , (c) 5.6 and (d) 4.5 each with an experimental error of \pm 0.1 . The solid line through the data shows the energy given by Eq. 5] The dotted and dashed lines show the energy given by the quasi-linear theory after one and two kicks respectively.

Our experimental setup has been detailed in other publications [6, 12]. Caesium atoms are typically cooled to below $10 \mu \mathrm{K}$ for our experiments in a standard MOT [13]. The atoms are then released from the trap and kicked twice by pulses from an optical standing wave detuned $500 \mathrm{MHz}$ from the $6 S_{1 / 2}(F=4) \rightarrow 6 P_{3 / 2}\left(F^{\prime}=5\right)$ transition of Caesium. The pulse width $\tau_{p}$ remained constant at $480 \mathrm{~ns}$ for all experimental runs. After a $12 \mathrm{~ms}$ expansion time, the atoms are effectively frozen in space by optical molasses and their resultant fluorescence is captured by a CCD camera giving information about the position distribution of the atoms after kicking. Knowing the time of flight of the atoms, we may infer their momentum distribution from the CCD image. We then find the energy of the atomic ensemble by numerically calculating the second moment of this distribution..

Fig. 2 shows experimental results after one and two kicks. For the results shown in this figure, $\kappa / \hbar$ was measured by finding the mean of the energies when $k \geq 1$ for both one and two kicks. The difference between these two values is given by the quasilinear energy growth rate $1 / 4(\kappa / \hbar)^{2}$. Using this parameter, and subtracting the thermal energy from both sets of results, we find good agreement between the analytical formula of Eq. [5 and the results obtained experimentally. Since the mean energy after one kick is expected to always be the trivial quasilinear result, the remainder of our results focus on the energy after two kicks. In this case, we measure $\sigma_{p}$ using a time of flight method and once again find the mean energy for the results where $k \geq 1$. We may then calculate $\kappa / \hbar$ using Eq. [4 In Fig. 3] the mean energy as a function of $k$ is shown for various values of the parameter $\kappa / \hbar$ corresponding to different laser powers used in our experiment. We notice in particular that the position in $k$ of the energy minimum and the point where deviation from the quasilinear result first occurs shift to the right as the value of $\kappa / \hbar$ is decreased. Additionally, it is interesting to note the value that the analytical expression tends to as $k \rightarrow 0$, even though this region was not able to be probed experimentally. In this limit, the energy due to kicking tends to $(\kappa / \hbar)^{2}$, which corresponds to ballistic energy growth with coefficient $1 / 4(\kappa / \hbar)^{2}$. Ballistic energy growth is usually associated with quantum resonance and, indeed, the $\hbar \rightarrow 0$ limit may be seen as a special case of quantum resonance. In fact, the $\epsilon-$ classical picture developed for the usual quantum resonances of the kicked rotor 14] should also be applicable in the regime near $k=0$. We anticipate investigating the energy peak as $k \rightarrow 0$ in more detail in the near future.

We have also investigated the effect of increasing $\sigma_{p}$ on the deviation from quasi-linear behaviour. We would expect that as $\sigma_{p}$ gets larger, the deviation from the quasi-linear energy in the second kick would become less prominent. To test this experimentally, we reduced the cooling efficiency of our MOT to create atomic samples with various momentum spreads of up to 6.02 -photon recoils. These samples were then kicked for the same value of $\kappa / \hbar$. As shown in Fig. 4 the deviations do indeed become less pronounced as the initial momentum spread is increased. However, modern kicked rotor experiments 


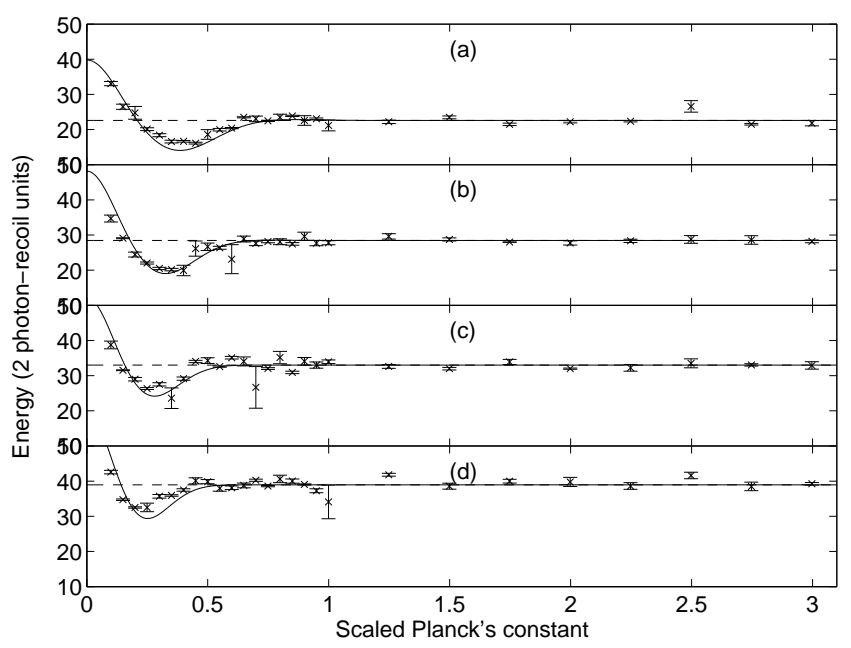

FIG. 4: Experimentally measured energies after two kicks for various values of $\sigma_{p}$. The measured values of $\sigma_{p}$ were (a) 3.3, (b) 4.2 , (c) 5.3 and (d) 6.0 in 2-photon recoils. We estimate the error in these measurements to be in the order of 0.92 photon recoil. For these curves, the measured value of $\kappa / \hbar$ is $5.8 \pm 0.2$. Solid lines show analytical results and dashed lines show the energy predicted by the quasi-linear theory.

typically achieve initial momentum distributions much narrower than 6 2-photon recoils, so experiments studying the early-time behaviour for small $k$ clearly need to take finite $-\sigma_{p}$ effects into account.

The structure seen in our results occurs for $k \lesssim 1$, where it seems reasonable to expect that the classical kicked rotor theory is of considerable relevance. In Fig. 5 the classical phase space distribution is shown for kicking periods corresponding to $k=0.001,0.3$ and 3.0 and values of $\kappa$ such that $\kappa / \hbar=5.5$. The trend in the momentum spread (and thus energy) as $k$ increases is apparent and does indeed appear to match that found in our measurements, in particular, the dip in energy seen near $k=0.3$. One factor governing this behaviour is the transition from regular motion to chaos as $\kappa$ is increased in the kicked rotor system. The onset of global chaos occurs for $\kappa \sim 1$ [15], corresponding to $k=0.18$ for the parameters used in Fig. 5] Thus the energy minimum would seem to correspond roughly to the transition to chaos where resonant motion has been destroyed but diffusive energy growth is still inhibited by KomolgorovArnold-Moser boundaries. We note that the phase space diagram was obtained using 100 iterations of the stan- dard map in order to emphasise the structure present. However, the qualitative behaviour of the system is seen to be the same as that after just two kicks.

In conclusion, we have presented new experimental results for the Atom Optics Kicked Rotor at very early times and as $k \rightarrow 0$. Although, the $\delta$-kicked approximation is no longer a good one for experiments in this regime, we nonetheless find excellent agreement between our measurements and recent analytical predictions. These results deviate significantly from the quasi-
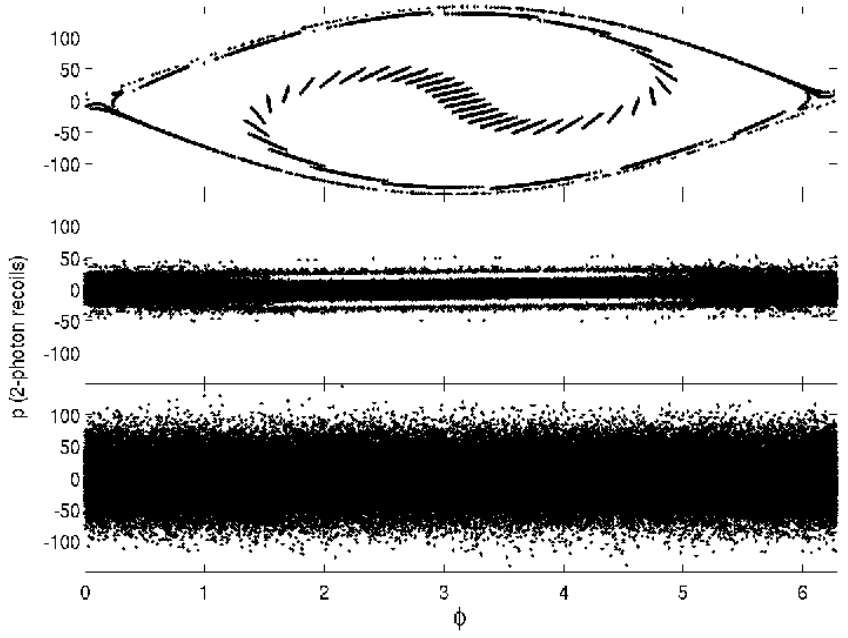

FIG. 5: Phase space diagrams for $T$ corresponding to $\hbar=$ (top) 0.001, (middle) 0.3 and (bottom) 3.0 and $\kappa$ such that $\kappa / \hbar=5.5$. The plots were generated from 100 iterations of the map in Eq. 2 using a uniform initial distribution in the $\phi$ coordinate and a Gaussian initial distribution in $p$ (momentum in experimental units) with $\sigma_{p}=3.6$.

linear energy growth in the first two kicks predicted by theories which assume a broad initial momentum distribution. The considerations raised in this report will be important for any future studies of the atom optics kicked rotor in the regime of small $k$. Additionally, the further study of this system for small $k$ is of interest as it relates to quantum resonance behaviour in the kicked rotor.

The authors would like to thank Sandro Wimberger for enlightening discussions about this work. M.S. is supported by a Top Achiever Doctoral Scholarship 03131. This work was supported by the Royal Society of New Zealand Marsden Fund, grant UOA016.
[1] F.L. Moore, et al., Phys. Rev. Lett. 754598 (1995).

[2] H. Ammann, R. Gray, I. Shvarchuck, and N. Christensen Phys. Rev. Lett, 80, 4111 (1998)

[3] J. Ringot, P. Szrifrgiser, J.C. Garreau and D. Delande Phys. Rev. Lett., 85, 2741 (2000)

[4] M.B. d'Arcy, et al., Phys. Rev. Lett. 87074102 (2001).

[5] C.F. Bharucha, et al., Phys. Rev. E, 60,3881 (1999)
[6] M.P. Sadgrove, et al., accepted for publication, Phys. Rev. E

[7] A.J. Daley and A.S. Parkins, Phys. Rev. E, 66, 056210 (2002)

[8] L. Deng, et al., Phys. Rev. Lett 835407 (1999)

[9] G. Duffy, et al., cond-mat/0401346

[10] A.B. Rechester and R.B. White, Phys. Rev. Lett. 441586 
(1980)

[11] D.L. Shepelyansky, Physica D 28, 103 (1987).

[12] M.E.K. Williams, et al., J. Opt. B 628 (2004).

[13] C. Monroe, W. Swann, H. Robinson and C. Wieman, Phys. Rev. Lett., 65, 1571 (1990)
[14] S. Wimberger, I. Guarneri, and S. Fishman, Nonlin. 16 1381 (2003).

[15] A.J. Lichtenberg, M.A. Lieberman Regular and chaotic dynamics, Springer-Verlag (1992) 